\title{
Stochastic allocation of virtual paths to ATM links
}

\author{
Michal Pióro and Piotr Gajowniczek \\ Institute of Telecommunications, Warsaw University of Technology, \\ Nowowiejska 15/19, 00-665 Warszawa, Poland \\ tel: +48 222598 20, fax: +4822 2549 50, e-mail: mpp@tele.pw.edu.pl
}

\begin{abstract}
In the paper we propose a way of solving the problem of allocating capacity of virtual paths to the links of an ATM network. We propose an effective stochastic method, called Simulated Allocation, which is able to find almost optimal allocation patterns in a short computation time. The method is simple to implement and can be used to the whole class of multicommodity flow problems, including the one considered in the paper. We present numerical examples illustrating effectiveness of the method.
\end{abstract}

Keyword Codes: G.1.6; I.6.1;

Keywords: Optimization; Simulation Theory

\section{Problem formulation}

The paper addresses the problem of allocation of virtual paths (VP, cf.[1],[2],[3]) to paths of the ATM network graph composed of ATM links with fixed capacity. Below we follow the problem formulation of [4].

Let $D$ be the set of traffic demands. Each traffic demand $d \in D$ is characterised by its:

- end nodes $v(d)$ and $w(d)$

- service class $s(d) \in S$

- required GOS, defined as the maximum allowed blocking probability $\beta(d)$ for the calls of $d$

- offered traffic $A(d)$ (defined as the call arrival intensity times the mean call holding time)

- set of admissible paths $P(d)$ for realising VPs for the demand in the considered multigraph $G$ of ATM links (one VP can be realised on each path $p \in P(d)$ ).

In the paper we assume that VPs are established for each origin-destination pair of nodes (OD pair) and for each service class, provided there is positive traffic. 
Let $E$ denote the set of links of graph $G$. Each link $e \in E$ has fixed capacity $c(e)$ expressed in capacity units. A capacity unit is defined as a rate [cells/sec] large enough to carry a call of any service class from the set of all service classes $S$. With each $s \in S$ there is associated a function $f_{s}(i)(i=0,1,2, \ldots)$ giving the maximum number of calls of class $s$ that can be supported by $i$ capacity units on a link, i.e. the number of virtual circuits supported by $i$ capacity units on one VP (cf.[5]). A feasible (flow allocation) state is defined by assigning for each traffic demand $d \in D$ the capacity $y(d, p)$ (expressed in capacity units) to its VP on each path $p \in P(d)$, so that the total capacity of the VPs of $d$, expressed in virtual circuits $C(d)=\Sigma_{p \in P(d)} f_{s(d)}(y(d, p))$, is sufficient to satisfy GOS, i.e.

$\mathscr{E}(C(d), A(d)) \leq \beta(d)$

and that for no edge $e$ the resulting number of capacity units allocated to it exceeds its capacity $c(e)(\mathscr{E}(\cdot, \cdot)$ denotes the Erlang loss formula).

The problem is to find a feasible state (flow allocation) maximizing the traffic carried in the network.

The formulated problem is a capacitated non-linear integer-valued multicommodity flow problem [6] and as such is $N P$-complete. For its solving we propose a stochastic allocation method, called Simulated Allocation (SA). The method was already presented in [7] in the context of the SDH network design. SA seems to be effective for solving integer-valued multicommodity flow problems, even if the allocation is "tight", i.e. when realisation of the demanded flow pattern requires almost all capacity of the network links. To allocate VPs we consider several allocation rules, from a simple random rule, through a natural "least-loaded path" rule, to a "traffic dependent" rule described in [8].

In the paper we compare the performance of SA with the method used in [9]. It follows from the results presented in Section 4 that SA, allowing for disconnection of already allocated VPs, gives a possibility of "overcoming" local minima of the objective function, allowing for achieving a reasonable solution in a reasonable time.

\section{Simulated Allocation}

Below we describe a version of SA for the capacitated multicommodity flow allocation problem formulated above. For each traffic demand $d \in D$ we compute (cf. Section 3) a minimum number $t(d)$ of virtual circuits such that

$\mathscr{E}(t(d), A(d)) \leq \beta(d)$

i.e. $t(d)$ is the minimum number of virtual circuits required to carry the traffic $A(d)$ with the required GOS. Then, with each traffic demand we associate a Poissonian stream of arrivals, called VP-demands, with intensity $\lambda(d, y)$ depending on state $y$ of the network (the notion of the state is described below). For each $d \in D$ a VP-demand arriving from its stream requires one capacity unit and is allocated (added) to the VP on an accessible path between $v(d)$ and $w(d)$ (i.e. on a path from $P(d)$ whose all edges have at least one free capacity unit). An arrival is rejected if no path is currently accessible. The essence of SA is that we allow the already realised VP-demands to be disconnected (with rate $\mu(d)$ for each VP-demand of traffic demand $d \in D$ ). 
The state space $Y$ of the introduced Markov chain is finite with the states of the form

$\boldsymbol{y}=\{y(d, p), d \in D, p \in P(d)\}$,

where $y(d, p)$ is the number of capacity units allocated to a VP of demand $d$ realised on path $p \in P(d)$. The state space $Y$ depends on the arrival intensities $\lambda(d, y)$ and on the rule of selecting accessible paths for allocation of arrivals. Such an allocation rule should take into account the impact of a capacity unit allocation on the value of the objective function (i.e. carried traffic). For reasonable allocation rules the characterisation of the state space is rather straightforward and the chain is irreducible. Let $x(d, y)$ denote the number of virtual circuits of traffic demand $d$ realised in state $y$, i.e. $x(d, y)=\Sigma_{p \in P(d)} f_{s(d)}(y(d, p))$. A state $y$ is said to be feasible if $x(d) \geq t(d)$ for all $d \in D$ and for no edge $e \in E$ its capacity $c(e)$ is exceeded. Below we assume that a feasible state exists.

In order to find an allocation pattern of VP-demands we generate a trajectory of the underlying Markov chain. The trajectory is terminated when for each traffic demand $d \in D$, $t(d)$ or more virtual circuits are allocated. If such a flow pattern cannot be found in a reasonable time (such a pattern may not exist at all) we may stop the process after certain time limit or by using some other (trajectory dependent) stopping rule.

Let us consider the imbedded discrete-time Markov chain (called allocation chain) with time epochs corresponding to arrival and disconnection instants of the original (continuous time) Markov chain implicitly described above. The number of steps to reach any feasible state (first passage time to the set of feasible states) from a fixed initial state is a random variable called allocation time and denoted by $T$. Notice that $T$ is finite with probability 1 , and so is its expected value. This last quantity is called allocation effort and is denoted by $F$. The value of $F$ for the considered problem depends on arrival intensities $\lambda(d, y)$, disconnection rates $\mu(d)$ and on the selection rule.

\section{Implementation of SA}

To generate trajectories of the allocation chain we have adapted the method used in [10] for simulation of circuit-switched networks with alternative routing. The version of SA described below requires that for all $d \in D \lambda(d, y))=\nu(y) \cdot(n(d)-k(d, y))$ for some statedependent intensity $\nu(y)$ and that $\mu(d)=1 . n(d)$ is defined as the minimum number such that

$$
\mathscr{E}\left(n(d) \cdot f_{s(d)}(1), A(d)\right) \leq \beta(d),
$$

i.e. $n(d)$ is equal to the minimum number of capacity units sufficient to carry the traffic demand under assumption that each of its VPs takes only one capacity unit (this is the worst case). $k(d, y)$ denotes the total number of capacity units allocated to demand $d$ (on all its admissible paths) in state $y$.

Let $M(y)$ denote the total number of VP-demands realised in state $y$. The information about the realised VP-demands is kept in the first $M(y)$ entries of a vector $A(\cdot)$, called the allocation vector, of fixed length $L$ equal to the total number of VP-demands, i.e.

$$
L=\Sigma_{d \in D} n(d) .
$$


Thus $L=M(y)+N(y)$ where $N(y)$ is the number of unrealised VP-demands in state $y$. The information about the unrealised VP-demands is kept in the remaining entries $M(y)+1, M(y)+2, \ldots, L$ of vector $A$. Each entry of the vector is a record identifying the traffic demand $d$ of the VP-demand and, if the VP-demand is currently realised, the path $p$ the VPdemand is realised on. In all states of the generated trajectory we keep the proportion of the total arrival rate $(\nu(y) \cdot N(y))$ to the total disconnection rate $(M(y))$ equal to a constant $\Lambda$. It follows that $\nu(y)=\Lambda \cdot M(y) / N(y)$.

Suppose that the simulated trajectory is in state $y$ in time epoch $i$. The state in time $i+1$ is determined using a random number $\xi$ from the interval $(0, M(y)+\nu(y) \cdot N(y))$.

Disconnection: $\xi \leq M(y)$.

Let $\eta=\operatorname{int}(\xi)+1$ (where int $(\cdot)$ denotes integer part). The VP-demand identified by record $A(\eta)$ is disconnected: the capacity units occupied by the VP-demand are released on edges of the path $p$ used by the VP-demand, records $A(\eta)$ and $A(M(y))$ are interchanged, and $M(y)$ is decremented by 1 .

Arrival: $\xi>M(y)$.

The integer

$\eta=\lfloor(\operatorname{int}(\xi)-M(y)-1) / \nu(y)\rfloor+M(y)+1$

identifies the arriving VP-demand in the allocation vector. If there currently exists an accessible path for the arriving VP-demand, one capacity unit on each edge of the selected path $p$ is seized, records $A(M(y)+1)$ and $A(\eta)$ are interchanged (the information about the selected path $p$ is added to record $A(\eta))$, and $M(y)$ is incremented by 1 . Otherwise, if there is no accessible path for the VP-demand, the arrival is rejected and the state $y$ and vector $A$ remain unaffected.

Note that the described method for generating trajectories of the allocation chain does not require any searching and thus is extremely fast.

For maximising the objective function (carried traffic) we use the following allocation rules:

- RAN: random choice from the set of accessible paths (used as the reference rule).

- LLP: least-loaded path: LLP searches for an accessible path maximising the minimum amount of free capacity (minimum taken over the edges of the path).

- MAX: the rule of [8]: the gain in terms of traffic carried on a VP due to adding a capacity module to it, minus the sum of (potential) carried traffics on all direct VPs on edges of the considered VP, that would be carried if one capacity unit were added to them.

\section{An example}

Below we present results of a study of the VP allocation problem for a 20 -node network originally considered in [9]. We compare the results of SA with the results obtained by a specialised method described in [9]. The method of [9] is a refinement of the method 
described in [4], which in turn is an extension (to the VP allocation problem) of the method proposed in [8] for SDH networks. It should perhaps be emphasised that the VP allocation method described in [4] and [9] is not the main goal of these two papers.

The considered network is depicted in Fig.1. It consists of 20 nodes and 42 edges with capacity varying from 26 to 623 capacity units. One service class $s$ is assumed, with $f_{s}(i) \equiv 10 \cdot i$ (one service unit supports 10 virtual circuits). The sets of admissible paths for OD pairs are predefined (several paths per OD pair).

Eight traffic patterns of all 190 traffic demands are considered. For all the patterns the total traffic offered is almost the same, but its distribution among individual traffic demands is different. Each traffic pattern contains traffic demands ranging from single Erlangs to over 300 Erlangs (detailed traffic patterns are given in Appendix).

Figure 1 20-node network graph with edge capacities

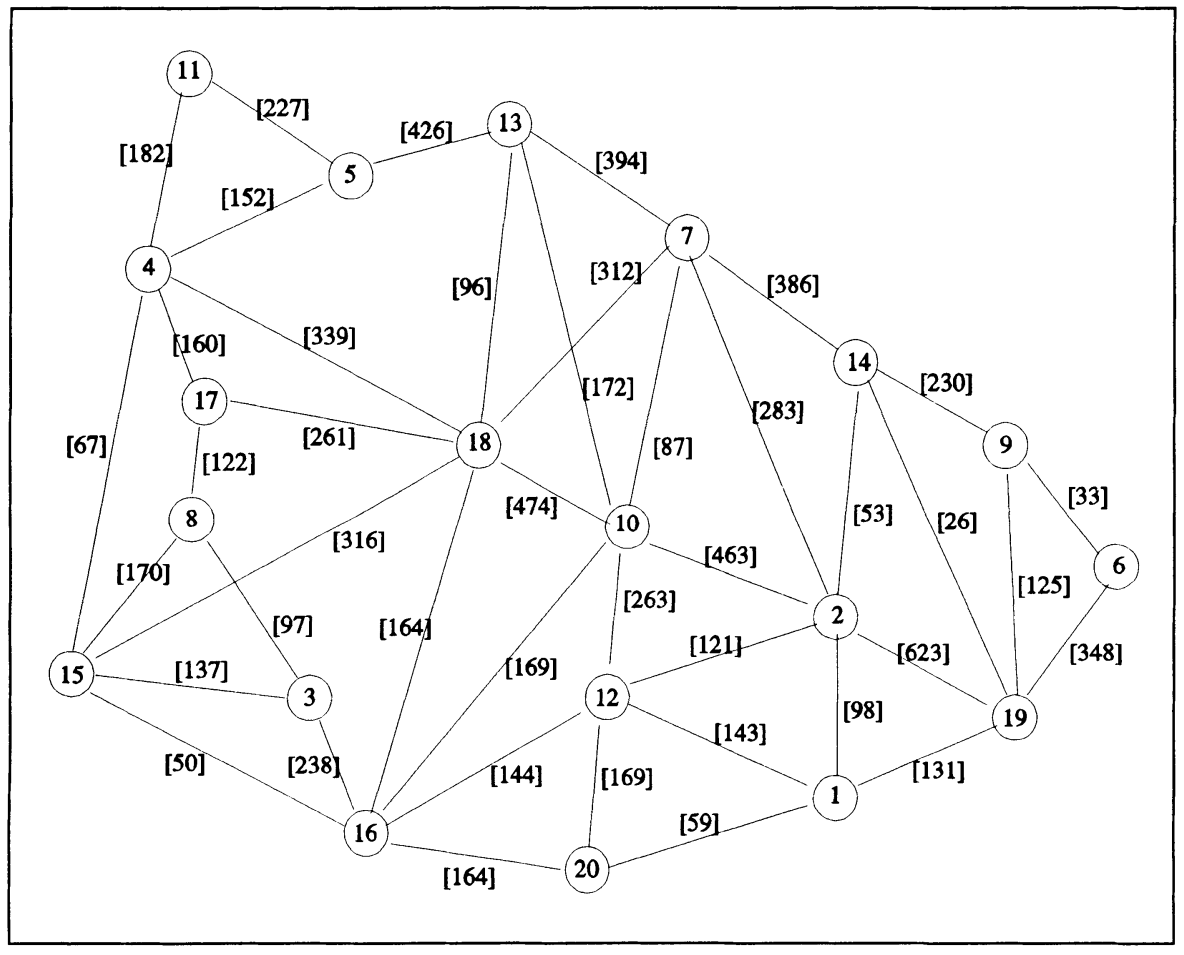

In the subsequent tables the results obtained with SA are compared with the results of the method of [9] for each traffic pattern. The results of SA, implemented in C under DOS, were computed on a PC $386 / 40 \mathrm{MHz}$ class computer. In all cases the trajectories were generated from scratch (from the idle state). The stopping rule is as follows. Let $\beta$ be the common blocking constraint (requirement) for all traffic demands, i.e. $\beta(d)=\beta$ for all $d \in D$ (in the example we assumed $\beta=2 \%$ ). First we set blocking threshold $\tau$ to $10 \cdot \beta$ and start to generate a trajectory (from scratch). When all individual blocking probabilities $b(d)$ become 
less than $\tau$ we change the threshold to $5 \cdot \beta$ and continue. If this threshold is reached we set $\tau$ to $3 \cdot \beta$, then to $2 \cdot \beta$, and finally to $\beta$. After reaching a consecutive blocking threshold the current solution is stored. If we cannot satisfy a consecutive threshold in a certain number of steps we stop the trajectory.

For each of the eight traffic patterns the results obtained from SA were averaged over 10 independent trajectories generated for each case, and are given with the $95 \%$ confidence interval. The sets of accessible paths for all OD pairs were predefined.

The notation used in the tables is as follows:

- TTC: total traffic carried

- TTO: total traffic offered

- $\mathrm{E}(\mathrm{B})$ : average blocking probability, i.e. the ratio of the total traffic lost to the total traffic offered: $\mathrm{E}(\mathrm{B})=(\mathrm{TTO}-\mathrm{TTC}) / \mathrm{TTO}$

- $\operatorname{Max}(\mathrm{B})$ : maximum of blocking probability (over all demands)

- F: allocation effort

- $\mathrm{T}_{\mathrm{m}}$ : mean simulation time.

It should be emphasised that in all eight traffic cases the network capacity was almost exhausted ( 97 to $99 \%$ of the network capacity was used to allocate VPs).

Table 1 Traffic pattern 1

\begin{tabular}{lllll}
\hline & RAN & LLP & MAX & method of [9] \\
\hline TTC & $28625.74 \pm 0.04 \%$ & $28916.01 \pm 0.03 \%$ & $28912.89 \pm 0.02 \%$ & 28823.24 \\
TTC/TTO & $0.98 \pm 0.04 \%$ & $0.99 \pm 0.03 \%$ & $0.99 \pm 0.02 \%$ & 0.98 \\
E(B)[\%] & $1.99 \pm 1.78 \%$ & $0.99 \pm 2.48 \%$ & $1.01 \pm 2.28 \%$ & 1.32 \\
$\operatorname{Max}(\mathrm{B})[\%]$ & $4.93 \pm 1.09 \%$ & $2.94 \pm 1.20 \%$ & $2.91 \pm 1.97 \%$ & 7.21 \\
F & $185234 \pm 19 \%$ & $136693 \pm 17 \%$ & $140993 \pm 18 \%$ & - \\
$\mathrm{T}_{\mathrm{m}}[\mathrm{s}]$ & 48.3 & 35.4 & 80.6 & - \\
\hline
\end{tabular}

Table 2 Traffic pattern 2

\begin{tabular}{lllll}
\hline & RAN & LLP & MAX & method of [9] \\
\hline TTC & $28117.17 \pm 0.05 \%$ & $28549.01 \pm 0.02 \%$ & $28544.76 \pm 0.02 \%$ & 28441.82 \\
TTC/TTO & $0.97 \pm 0.05 \%$ & $0.99 \pm 0.02 \%$ & $0.99 \pm 0.02 \%$ & 0.98 \\
E(B)[\%] & $2.52 \pm 1.92 \%$ & $1.02 \pm 1.83 \%$ & $1.01 \pm 2.28 \%$ & 1.39 \\
$\operatorname{Max}(B)[\%]$ & $5.90 \pm 0.7 \%$ & $2.97 \pm 0.99 \%$ & $2.99 \pm 0.26 \%$ & 6.73 \\
F & $132258 \pm 11 \%$ & $100411 \pm 19 \%$ & $179480 \pm 21 \%$ & - \\
$\mathrm{T}_{\mathrm{m}}[\mathrm{s}]$ & 32.0 & 23.5 & 107.5 & - \\
\hline
\end{tabular}

Table 3 Traffic pattern 3

\begin{tabular}{lllll}
\hline & RAN & LLP & MAX & method of [9] \\
\hline TTC & $28142.24 \pm 0.04 \%$ & $28422.76 \pm 0.03 \%$ & $28430.42 \pm 0.02 \%$ & 28311.00 \\
TTC/TTO & $0.98 \pm 0.04 \%$ & $0.99 \pm 0.03 \%$ & $0.99 \pm 0.02 \%$ & 0.99 \\
E(B)[\%] & $1.88 \pm 1.94 \%$ & $0.90 \pm 2.85 \%$ & $0.87 \pm 2.79 \%$ & 1.29 \\
$\operatorname{Max}(B)[\%]$ & $4.90 \pm 1.26 \%$ & $2.92 \pm 1.51 \%$ & $2.86 \pm 1.56 \%$ & 8.61 \\
F & $82947 \pm 4 \%$ & $23215 \pm 8 \%$ & $34409 \pm 5 \%$ & - \\
$\mathrm{T}_{\mathrm{m}}[\mathrm{s}]$ & 21.3 & 6.6 & 34.7 & - \\
\hline
\end{tabular}


Table 4 Traffic pattern 4

\begin{tabular}{lllll}
\hline & RAN & LLP & MAX & method of [9] \\
\hline TTC & $28800.27 \pm 0.02 \%$ & $29180.32 \pm 0.03 \%$ & $29169.73 \pm 0.02 \%$ & 29123.32 \\
TTC/TTO & $0.97 \pm 0.02 \%$ & $0.98 \pm 0.03 \%$ & $0.98 \pm 0.02 \%$ & 0.98 \\
E(B)[\%] & $2.73 \pm 0.71 \%$ & $1.44 \pm 1.96 \%$ & $1.48 \pm 1.43 \%$ & 1.64 \\
$\operatorname{Max}(B)[\%]$ & $5.93 \pm 0.74 \%$ & $3.93 \pm 1.05 \%$ & $3.95 \pm 0.37 \%$ & 8.63 \\
F & $230836 \pm 38 \%$ & $67860 \pm 8 \%$ & $146730 \pm 22 \%$ & - \\
$\mathrm{T}_{\mathrm{m}}[\mathrm{s}]$ & 55.1 & 16.2 & 92.2 & - \\
\hline
\end{tabular}

Table 5 Traffic pattern 5

\begin{tabular}{lllll}
\hline & RAN & LLP & MAX & method of [9] \\
\hline TTC & $27824.75 \pm 0.03 \%$ & $28098.95 \pm 0.04 \%$ & $28111.16 \pm 0.03 \%$ & 29062.24 \\
TTC/TTO & $0.98 \pm 0.03 \%$ & $0.99 \pm 0.04 \%$ & $0.99 \pm 0.03 \%$ & 0.99 \\
E(B)[\%] & $1.85 \pm 2.60 \%$ & $0.88 \pm 4.40 \%$ & $0.84 \pm 3.98 \%$ & 1.01 \\
$\operatorname{Max}(B)[\%]$ & $4.90 \pm 1.26 \%$ & $2.87 \pm 4.63 \%$ & $2.86 \pm 3.77 \%$ & 5.57 \\
F & $69670 \pm 4 \%$ & $16829 \pm 15 \%$ & $33195 \pm 7 \%$ & - \\
$\mathrm{T}_{\mathrm{m}}[\mathrm{s}]$ & 17.7 & 4.2 & 34.5 & - \\
\hline
\end{tabular}

Table 6 Traffic pattern 6

\begin{tabular}{lllll}
\hline & RAN & LLP & MAX & method of [9] \\
\hline TTC & $28185.40 \pm 0.02 \%$ & $28421.27 \pm 0.03 \%$ & $28423.21 \pm 0.02 \%$ & 28426.80 \\
TTC/TTO & $0.99 \pm 0.02 \%$ & $0.99 \pm 0.03 \%$ & $0.99 \pm 0.02 \%$ & 0.99 \\
E(B)[\%] & $1.38 \pm 1.36 \%$ & $0.56 \pm 4.60 \%$ & $0.55 \pm 3.18 \%$ & 0.54 \\
$\operatorname{Max}(B)[\%]$ & $3.90 \pm 0.60 \%$ & $1.93 \pm 1.55 \%$ & $1.96 \pm 1.63 \%$ & 2.0 \\
F & $119578 \pm 4 \%$ & $175202 \pm 23 \%$ & $177987 \pm 21 \%$ & - \\
$\mathrm{T}_{\mathrm{m}}[\mathrm{s}]$ & 30.9 & 44.8 & 95.6 & - \\
\hline
\end{tabular}

Table 7 Traffic pattern 7

\begin{tabular}{lllll}
\hline & RAN & LLP & MAX & method of [9] \\
\hline TTC & $28650.90 \pm 0.04 \%$ & $29228.89 \pm 0.02 \%$ & $29219.84 \pm 0.03 \%$ & 29121.92 \\
TTC/TTO & $0.96 \pm 0.04 \%$ & $0.98 \pm 0.02 \%$ & $0.98 \pm 0.03 \%$ & 0.97 \\
E(B)[\%] & $3.88 \pm 0.87 \%$ & $1.94 \pm 1.19 \%$ & $1.97 \pm 1.52 \%$ & 2.3 \\
$\operatorname{Max}(B)[\%]$ & $7.84 \pm 0.41 \%$ & $4.85 \pm 0.89 \%$ & $4.78 \pm 1.80 \%$ & 8.22 \\
F & $166185 \pm 13 \%$ & $91012 \pm 14 \%$ & $178537 \pm 19 \%$ & - \\
$\mathrm{T}_{\mathrm{m}}[\mathrm{s}]$ & 40.3 & 20.9 & 98.9 & - \\
\hline
\end{tabular}

Table 8 Traffic pattern 8

\begin{tabular}{lllll}
\hline & RAN & LLP & MAX & method of [9] \\
\hline TTC & $28774.35 \pm 0.05 \%$ & $29060.29 \pm 0.03 \%$ & $29061.17 \pm 0.02 \%$ & 28903.74 \\
TTC/TTO & $0.98 \pm 0.05 \%$ & $0.99 \pm 0.03 \%$ & $0.99 \pm 0.02 \%$ & 0.98 \\
E(B)[\%] & $1.91 \pm 2.43 \%$ & $0.94 \pm 2.96 \%$ & $0.94 \pm 2.00 \%$ & 1.47 \\
$\operatorname{Max}(\mathrm{B})[\%]$ & $4.92 \pm 0.98 \%$ & $2.95 \pm 1.23 \%$ & $2.87 \pm 3.12 \%$ & 7.78 \\
$\mathrm{~F}$ & $117435 \pm 6 \%$ & $59209 \pm 14 \%$ & $70670 \pm 11 \%$ & - \\
$\mathrm{T}_{\mathrm{m}}[\mathrm{s}]$ & 29.6 & 14.9 & 50.0 & - \\
\hline
\end{tabular}


The subsequent figures show the distribution of traffic carried (in percents of the total traffic carried) with respect to the blocking probability level (more precisely: the curves show how many percents of traffic is carried with blocking probability greater than $B$. Figures 1 through 8 show the distributions for the allocation rule LLP.

Figure 2 Traffic pattern 1

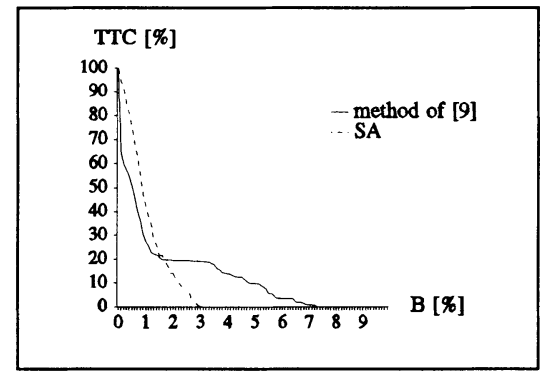

Figure 4 Traffic Pattern 3

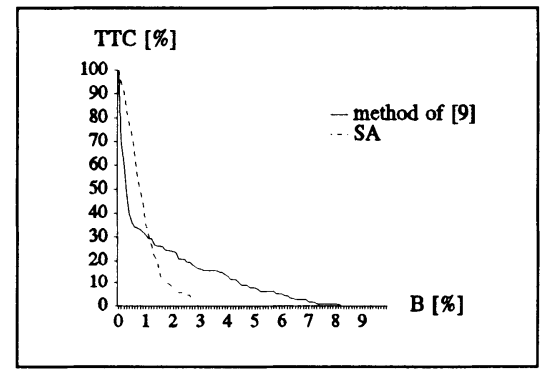

Figure 6 Traffic pattern 5

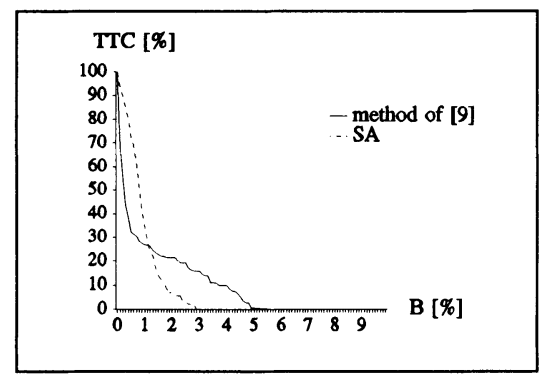

Figure 3 Traffic pattern 2

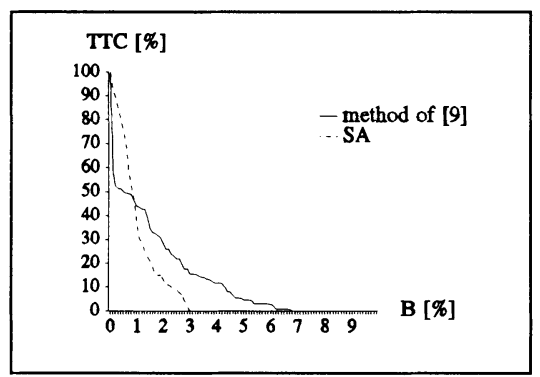

Figure 5 Traffic pattern 4

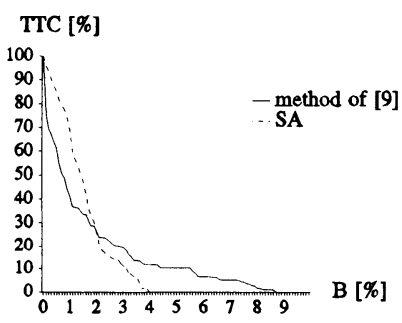

Figure 7 Traffic pattern 6

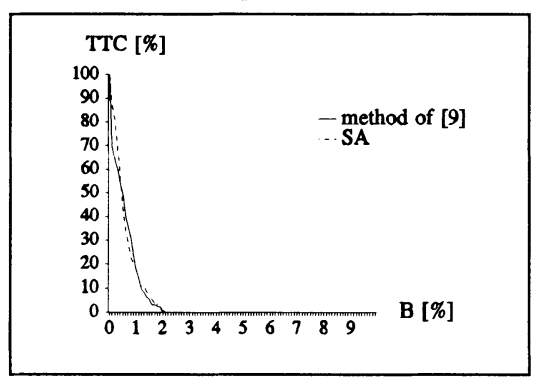


Figure 8 Traffic pattern 7

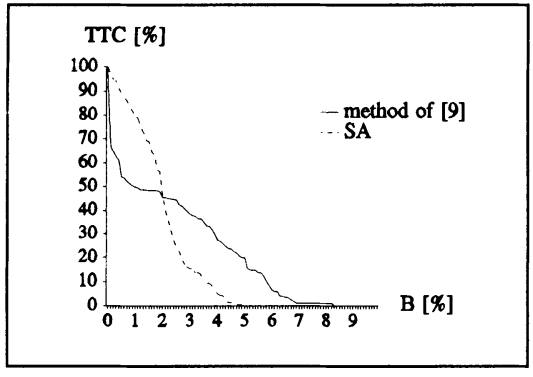

Figure 9 Traffic pattern 8

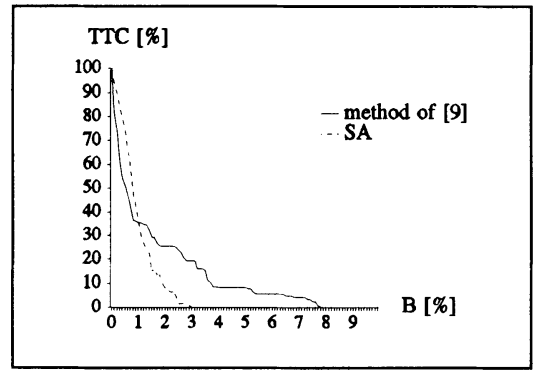

The following conclusions may be derived from the results presented in this section:

- The rules LLP and MAX find practically equivalent solutions in terms of carried traffic TTC and maximum individual blocking Max(B).

- In most cases LLP indicates considerably less allocation effort $F$ to find the solution than MAX. In 4 cases LLP needs half of the effort of MAX (traffic patterns 2,4,5,7), in 2 cases several tens of percents less (patterns 3,8), and in two cases the effort of LLA is only slightly less (patterns 1,6 ).

- The computation time of LLP is even more considerably less than for MAX, because the LLA rule is much faster than MAX.

- Solutions yielded by the random rule RAN are clearly worse than those of LLP and MAX, although not significantly (TTC is less by $2 \%$ in the worst case of pattern 7). Maximum individual blocking for RAN is typically 1.5 to 2 times greater.

- Allocation effort for RAN is in most cases, except for traffic pattern 6, significantly greater than for LLA. Thus, even that the allocation rule RAN is faster and its solutions are worse than those of LLP, the latter needs significantly less computation times.

- Out of the three considered rules, LLP is clearly the most effective, both in terms of the solution quality and computation time (allocation effort).

- The maximum TTC found by the method of [9] is typically slightly less than for SA with SA/LLP or SA/MAX, and slightly better than for SA/RAN.

- The greatest difference between the two approaches is that the method of [9] distributes losses much less evenly than SA. This is illustrated in figures 1-8. Only for the traffic pattern 6 both methods are almost equivalent. For the rest of the patterns SA/LLP has clearly much shorter distribution tail.

\section{Final remarks}

As illustrated in Section 4 the Simulated Allocation (SA) method is effective for the considered problem of allocation of virtual paths to ATM links of fixed capacity. The method is very simple to implement and is of general character (SA was already used in almost the same form for the problem of allocating of trunk groups to SDH transmission network).

Numerical invistigations (partly reported in [7]) show that the allocation effort, and thus computation time, of SA is linear with respect to the number of demanded modules (VP-demands) and to the number of the network nodes. 
SA is not too sensitive to the choice of the allocation rule, as illustrated in Section 4 and in [7]. So far, for the considered type of the capacitated allocation multicommodity flow problems, we have found the "least-loaded path" allocation principle to be the most effective, both in terms of the solution quality and the computation time.

It seems that crucial to effectiveness of SA is the choice of sets of admissible paths for OD pairs. So far we have used predefined sets of paths found by other methods or a separate procedure. A natural extension of SA would be to let it search for an optimum (shortest for a suitable edge metric) path dynamically, while handling an arrival event. This does not have to be done for each arrival (a set of admissible paths can be updated e.g. only if the current paths are not accessible).

Finally, let us notice that other, service-specific GOS measures, besides the call blocking probability used in this paper, may be assumed by SA for the allocation criterion. These other measures may be adopted for instance for services whose calls may wait for access to an ATM path. To achieve this, other functions than the Erlang loss formula would have to be considered.

\section{Acknowledgement}

The authors wish to thank Dr.A..Arvidsson, Lund Institute of Technology, Sweden for providing the network example used in this paper and with detailed results of the network optimisation by his method.

\section{References}

1. Addie, R. and Warfield, R.: "Bandwidth Switching and New Network Architectures", Proc. of the 12th ITC, Torino, June, 1988.

2. Sato, K-I., Ohta, S. and Tokizawa, I.: "Broad-Band ATM Network Architecture Based on Virtual Paths", IEEE Trans. on Communications, vol.38, No.8, 1990.

3. Ohta, S. and Sato, K-I.: "Dynamic Bandwidth Control of the Virtual Path in an ATM Network", IEEE Trans. on Communications, vol.40, No.7, 1992.

4. Arvidsson, A.: "Management of Reconfigurable Virtual Path Networks", Proc. of the 14th ITC, Antibes, June, 1994.

5. Guérin, R., Ahmadi, H. and Naghshineh, M.: "Equivalent Capacity and its Applications to Bandwidth Allocation in High-Speed Networks, IEEE J.Sel. Areas in Commun., vol.9, No.7, 1991.

6. Assad, A.A.: "Multicommodity Network Flows - A Survey", Networks, Vol.8 (1978).

7. Pióro, M. and Gajowniczek, P.: "Simulated Allocation - a Suboptimal Solution to the Multicommodity Flow Problems", The 11th UK Teletraffic Symposium, Cambridge, March, 1994.

8. Gopal, G., Kim, C. and Weinrib, A.: "Algorithms for Reconfigurable Networks", Proc. of the 13th ITC, Copenhagen, June, 1991.

9. Arvidsson, Å.: "High Level B-ISDN/ATM Traffic Management in Real Time", Second Workshop on Performance Modelling and Evaluation of ATM Networks, IFIP TC6, Bradford, July, 1994.

10. Pióro, M., Reneby, L. and Wallstroem, B.: "Routing Principles in Non-Hierarchical Networks", Technical Report, Lund Institute of Technology, Lund, 1984. 


\section{Appendix:}

Traffic pattern 1:

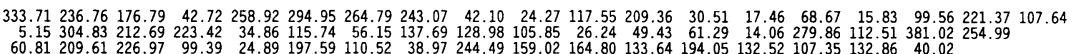

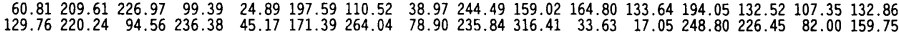

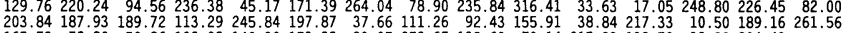

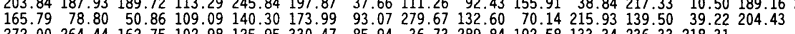

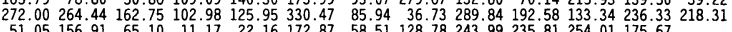

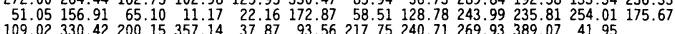

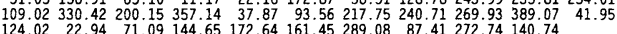

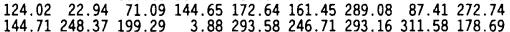

$\begin{array}{rrrrrrrr}144.71 & 248.37 & 199.29 & 3.88 & 293.58 & 246.71 & 293.16 & 311.58 \\ 210.93 & 81.99 & 189.85 & 62.99 & 32.80 & 192.34 & 159.73 & 212.28\end{array}$

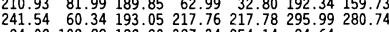

$\begin{array}{lllll}24.02 & 102.89 & 182.20 & 327.34 & 254.14\end{array}$

$\begin{array}{rrrrr}24.02 & 28.89 & 25.14 & 13.45 & 30.79\end{array}$

$\begin{array}{rrrr}24.84 & 3.60 & 33.05 & 282.28 \\ 182.72 & 239.57 & 73.81\end{array}$

122.72

Traffic pattern 2:

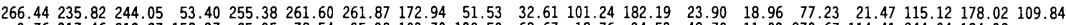

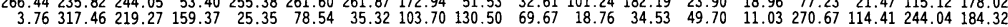

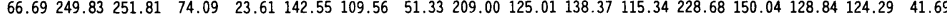

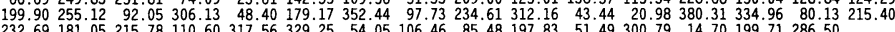

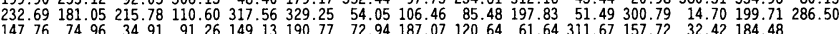

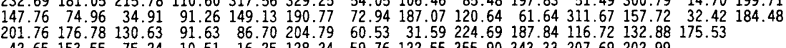

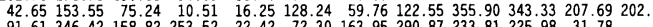

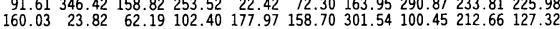

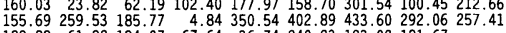

$\begin{array}{llrlllll}189.89 & 61.92 & 194.07 & 67.64 & 36.74 & 240.23 & 123.02 & 181.67\end{array}$

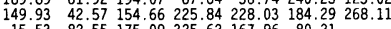

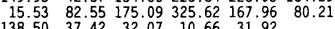

$138.50 \quad 37.42 \quad 32.07 \quad 10.66 \quad 31.92$

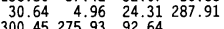

125.67345 .61

\section{Traffic pattern 3 :} $\begin{array}{rrrrrrrrrrrrrrrrrrrrrrrrr}321.45 & 300.03 & 262.15 & 54.15 & 316.98 & 289.38 & 320.08 & 250.27 & 72.79 & 28.54 & 167.02 & 245.36 & 29.69 & 20.45 & 103.37 & 22.72 & 107.25 & 221.05 & 166.76 \\ 4.40 & 264.95 & 184.66 & 181.69 & 22.04 & 84.31 & 44.28 & 120.50 & 104.66 & 92.10 & 15.68 & 35.72 & 49.94 & 13.28 & 261.48 & 94.82 & 241.35 & 261.12 & \end{array}$

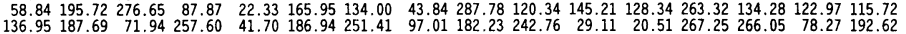

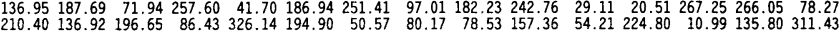

$\begin{array}{llllllllllllll}129.73 & 71.13 & 38.81 & 124.22 & 126.66 & 256.71 & 72.34 & 196.77 & 139.98 & 84.45 & 229.99 & 142.68 & 33.68 & 262.85\end{array}$

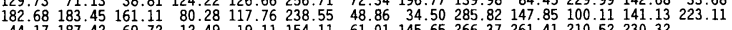

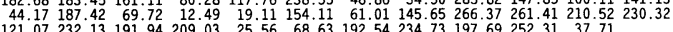

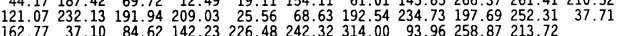

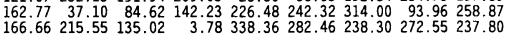

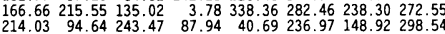

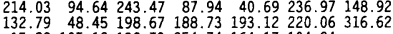

$\begin{array}{lllllll}15.82 & 105.18 & 132.73 & 254.74 & 164.17 & 104.84\end{array}$

$\begin{array}{lllll}156.86 & 30.26 & 26.60 & 10.51 & 41.11\end{array}$

$\begin{array}{rrrr}30.25 & 5.30 & 27.96 & 431.85\end{array}$

$172.3820999 \quad 93.88$

114.59319 .03

Traffic pattern 4:

$\begin{array}{llllllllllllllllllllll}254.18 & 233.95 & 231.10 & 44.97 & 248.28 & 317.17 & 256.02 & 180.14 & 46.74 & 30.67 & 95.99 & 218.18 & 23.08 & 17.56 & 76.74 & 19.59 & 122.81 & 177.70 & 114.24\end{array}$

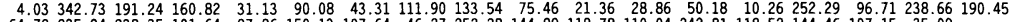
$\begin{array}{lllllllllllllllll}64.72 & 235.94 & 238.35 & 101.64 & 27.26 & 150.13 & 107.64 & 46.27 & 252.28 & 144.99 & 118.78 & 110.04 & 242.81 & 118.52 & 144.46 & 107.15 & 35.09\end{array}$

$\begin{array}{llllllllllllllll}207.09 & 273.72 & 104.62 & 268.20 & 44.92 & 194.72 & 402.95 & 96.30 & 283.31 & 303.65 & 38.93 & 20.05 & 276.47 & 374.56 & 94.00 & 209.06\end{array}$

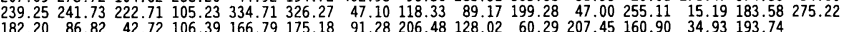

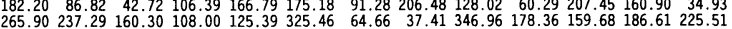

$\begin{array}{llllllllllll}35.77 & 146.82 & 79.86 & 11.83 & 21.97 & 144.73 & 48.52 & 110.09 & 243.99 & 305.78 & 213.35 & 210.48\end{array}$

$\begin{array}{ccccccccccc}103.66 & 313.27 & 150.61 & 282.83 & 22.51 & 64.96 & 179.94 & 231.73 & 259.89 & 278.64 & 35.59\end{array}$

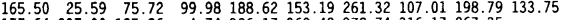

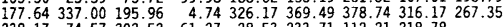

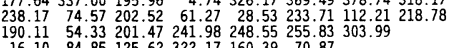

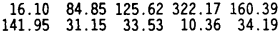

$\begin{array}{rrrr}141.95 & 31.15 & 33.53 & 10.36 \\ 23.65 & 4.63 & 25.42 & 299.18\end{array}$

240.94195 .21

130.88
88.31 
Traffic pattern 5:

$\begin{array}{rrrrrrrrrrrrrrrrrrrrrr}370.33 & 298.15 & 249.20 & 45.72 & 309.88 & 283.81 & 314.24 & 257.48 & 68.00 & 32.81 & 161.76 & 236.18 & 28.88 & 23.43 & 85.29 & 20.84 & 114.94 & 220.72 & 171.16 \\ 4.67 & 355.36 & 197.82 & 226.32 & 34.02 & 117.33 & 52.27 & 154.09 & 135.06 & 117.42 & 22.66 & 38.11 & 62.24 & 12.51 & 302.24 & 98.60 & 303.11 & 316.41\end{array}$

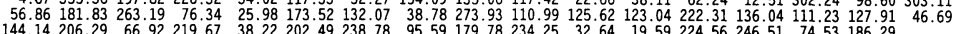

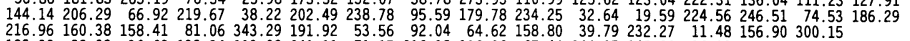

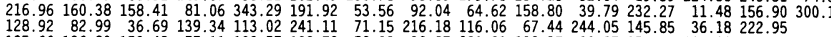

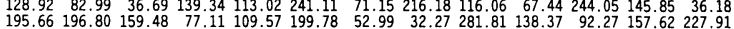

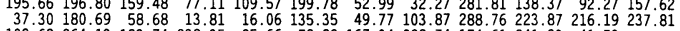

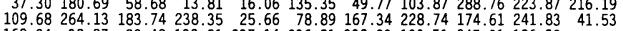

$\begin{array}{llllllllll}168.24 & 38.87 & 82.48 & 139.81 & 237.14 & 236.81 & 338.93 & 100.51 & 245.01 & 186.88\end{array}$

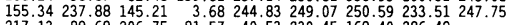

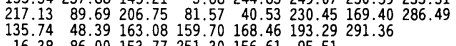

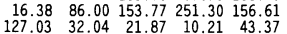

$\begin{array}{rrrr}127.03 & 32.04 & 21.87 & 10.21 \\ 23.26 & 3.96 & 22.88 & 373.96\end{array}$

$211.19 \begin{array}{rrr}23.96 & 22.82 & 96.36\end{array}$

119.80333 .03

123.71

Traffic pattern 6:

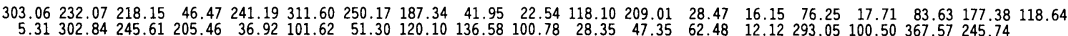

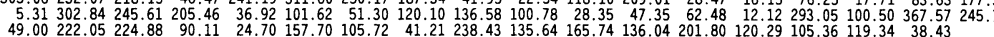

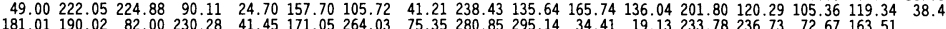

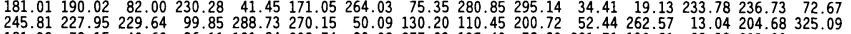

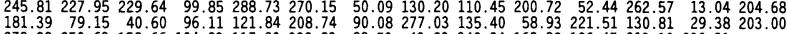

$\begin{array}{rrrrrrrrrrrrr}181.39 & 79.15 & 40.60 & 96.11 & 121.84 & 208.74 & 90.08 & 277.03 & 135.40 & 58.93 & 221.51 & 130.81 & 29.38 \\ 278.88 & 250.63 & 158.66 & 104.83 & 117.20 & 339.83 & 82.53 & 43.23 & 342.94 & 168.88 & 126.45 & 203.10 & 230.31\end{array}$

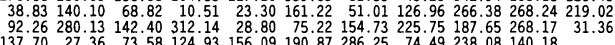

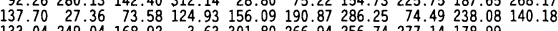

$\begin{array}{lllllllll}133.04 & 249.04 & 168.92 & 3.63 & 301.80 & 266.94 & 256.74 & 277.14 & 178.99\end{array}$

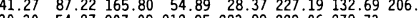

21.05108 .63181 .91318 .73194 .02100 .61

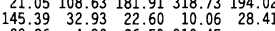

$\begin{array}{rrrr}22.86 & 4.30 & 26.53 & 310.45\end{array}$

$\begin{array}{ll}181.44 & 216.78 \\ 108.73 & 239.30\end{array}$

88.81

Traffic pattern 7:

$\begin{array}{lllllllllllllllllllllllll}235.79 & 231.13 & 236.25 & 47.22 & 237.64 & 217.10 & 308.39 & 215.52 & 51.38 & 30.87 & 101.79 & 181.83 & 21.86 & 22.03 & 67.21 & 23.35 & 122.63 & 177.22 & 120.84\end{array}$

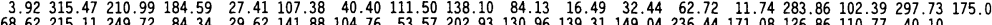

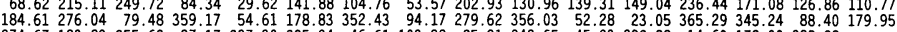

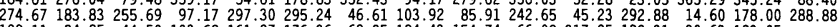

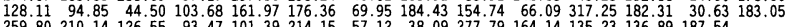

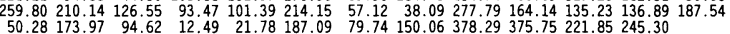

$\begin{array}{lllllllllllll}50.28 & 173.97 & 94.62 & 12.49 & 21.78 & 187.09 & 79.74 & 150.06 & 378.29 & 375.75 & 221.85 & 245.30\end{array}$

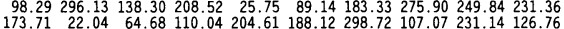

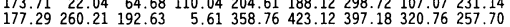

$\begin{array}{llllllll}175.06 & 67.16 & 215.20 & 59.54 & 40.37 & 275.08 & 127.28 & 176.12\end{array}$

$138.6960 .15168 .70221 .02 \quad 234.15215 .67266 .10$

$\begin{array}{rrrrr}21.34 & 88.29 & 174.80 & 317.01 & 149.04 \\ 163.75 & 49.93 & 35.73 & 12.55 & 37.59\end{array}$

$\begin{array}{rrrrr}28.67 & 4.65 & 23.99 & 246.93\end{array}$

$\begin{array}{rrr}28.67 & 4.65 & 23.99 \\ 299.16 & 253.15 & 81.24\end{array}$

125.01347 .02

Traffic pattern 8 :

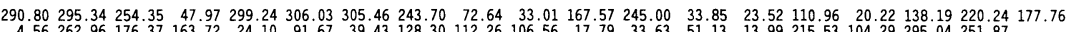

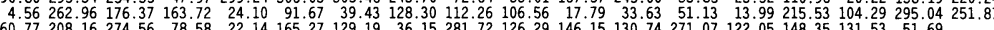

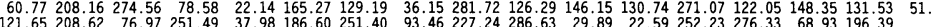

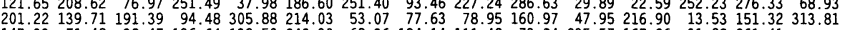

$\begin{array}{llllllllllllll}145.33 & 71.48 & 38.47 & 136.64 & 139.50 & 242.30 & 69.36 & 194.14 & 111.48 & 73.24 & 235.57 & 167.26 & 31.88 & 261.41\end{array}$

$\begin{array}{llllllllllllll}189.56 & 169.64 & 157.03 & 82.12 & 109.01 & 194.76 & 59.18 & 32.95 & 338.93 & 124.15 & 118.62 & 145.13 & 235.11\end{array}$

$\begin{array}{llllllllllll}41.88 & 170.60 & 57.77 & 14.46 & 15.86 & 142.46 & 53.51 & 143.83 & 288.76 & 293.83 & 175.53 & 225.46\end{array}$

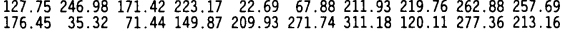

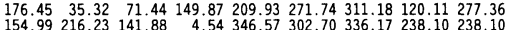

$\begin{array}{rrrrrrrr}154.99 & 216.23 & 141.88 & 4.54 & 346.57 & 302.70 & 336.17 & 238.10 \\ 244.38 & 82.28 & 219.42 & 95.51 & 36.26 & 322.97 & 153.18 & 292.98\end{array}$

$\begin{array}{lllllll}158.78 & 42.38 & 212.70 & 183.92 & 244.41 & 202.29 & 314.61\end{array}$

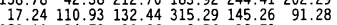

$\begin{array}{ccccc}182.11 & 34.71 & 30.26 & 9.76 & 38.73\end{array}$

$\begin{array}{rrrr}28.28 & 6.01 & 33.84 & 390.86\end{array}$

141.30
112.787 .59 